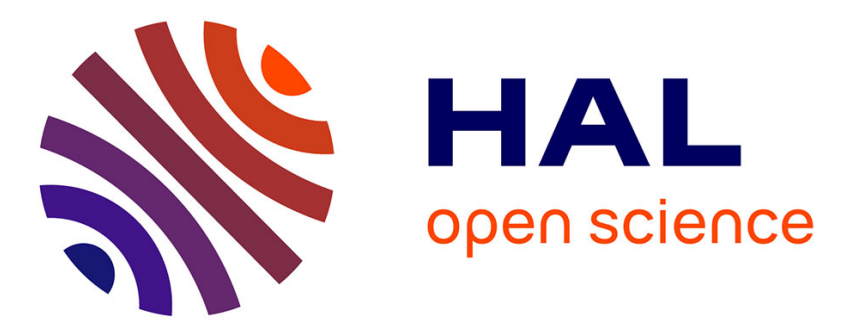

\title{
Towards an explanation of age-related difficulties in crossing a two-way street
}

\author{
Aurélie Dommes, Tristan Le Lay, Fabrice Vienne, Nguyen-Thong Dang, \\ Alexandra Perrot Beaudoin, Manh Cuong Do
}

\section{- To cite this version:}

Aurélie Dommes, Tristan Le Lay, Fabrice Vienne, Nguyen-Thong Dang, Alexandra Perrot Beaudoin, et al.. Towards an explanation of age-related difficulties in crossing a two-way street. Accident Analysis \& Prevention, 2015, 85, pp.229-238. 10.1016/j.aap.2015.09.022 . hal-01267164

\section{HAL Id: hal-01267164 \\ https://hal.science/hal-01267164}

Submitted on 4 Feb 2016

HAL is a multi-disciplinary open access archive for the deposit and dissemination of scientific research documents, whether they are published or not. The documents may come from teaching and research institutions in France or abroad, or from public or private research centers.
L'archive ouverte pluridisciplinaire HAL, est destinée au dépôt et à la diffusion de documents scientifiques de niveau recherche, publiés ou non, émanant des établissements d'enseignement et de recherche français ou étrangers, des laboratoires publics ou privés. 
To cite this article : Dommes, A., Le Lay, T., Vienne, F., Dang, N.-T., Perrot Beaudoin, A., Do, M.C. (2015). Towards an explanation of age-related difficulties in crossing a two-way street. Accident Analysis \& Prevention, 85, 229-238.

Towards an explanation of age-related difficulties in crossing a two-way street

Running head: Two-Way Street-Crossing and Ageing

Aurélie Dommes ${ }^{1}$, Tristan Le Lay ${ }^{1}$, Fabrice Vienne ${ }^{1}$, Nguyen-Thong Dang ${ }^{1}$, Alexandra Perrot Beaudoin ${ }^{2}$, Manh Cuong $\mathrm{Do}^{2}$

1. IFSTTAR-LEPSIS, Versailles, France

2. University Paris-Sud, UFR STAPS, CIAMS, Orsay, France

Corresponding author: Aurélie Dommes

IFSTTAR-LEPSIS

25 allée des Marronniers

78000 Versailles France

Phone: +33(0)130843943

E-mail: aurelie.dommes@ifsttar.fr 


\begin{abstract}
Crossing a two-way street is a complex task that involves visual, cognitive and motor abilities, all of which are known to decline with ageing. In particular, older pedestrians may experience difficulties when crossing two-way streets because of incorrect gap acceptance choices and impossible or unperceived evasive actions. To understand the overrepresentation of older pedestrians in crash statistics, several experimental studies have sought to identify traffic-related factors as well as those related to the abilities of the individuals themselves. However, none of these studies has required participants to actually walk across an experimental two-way street with curbs, which is a particularly challenging situation for older pedestrians. To fill this research gap, a quasi- experiment was conducted in a simulator including a total of 58 healthy aged participants (25 younger-old [age 60 - 72] and 33 olderold [age 72 - 92]) and 25 young adults (aged 18 - 25 years). Participants carried out a streetcrossing task in a simulated two-way traffic environment; curbs were present on both sides of the experimental street. Participants also undertook a battery of tests to assess their visual and cognitive abilities. In addition, during the experiment, the participants' gait parameters were recorded. In line with earlier findings, the older-old group of participants made a higher number of decisions that led to collisions with approaching cars compared with the other groups. The two groups of older participants experienced specific difficulties when vehicles were in the far lane or when they approached rapidly. A regression analysis identified visual acuity, speed of processing (assessed using the $\mathrm{UFOV}^{\circledR}$ test), and step length as significant predictors of collisions. Our results have implications for understanding the difficulties experienced by older pedestrians and allow to draw up several recommendations for improving their safety.
\end{abstract}

Keywords : Ageing, Street-crossing, Collisions, Vision, Cognition, Mobility 


\section{Introduction}

More than half of all pedestrians killed on the roads in France are over 65 years of age (indeed, the same is true in several European countries); however, this age group represents less than $17 \%$ of the French population (ONISR, 2012). The overrepresentation of older pedestrians in crash statistics is often explained by their greater frailty, older people generally recover less well from physical injuries. Walking is also often reported to be their preferred mode of transport in urban cities, leading to greater exposure effects. But slower walking speeds and altered decision-making processes have also been reported with ageing (see e.g., Dommes et al., 2014; Holland and Hill, 2010; Oxley et al, 2005). Since the 1990s, these wellknown, if somewhat worrying figures have actually motivated an increasing number of studies to determine which characteristics of infrastructure, traffic and/or pedestrians are likely to increase the risk of being involved in an accident with ageing.

Observational studies (Oxley et al., 1997; Zhuang and Wu, 2011, 2012) and accident analyses (Fontaine and Gourlet, 1997; Dunbar, 2012) have shown that ageing brings greater difficulties in crossing the street especially in complex traffic situations such as two-way streets. On the other hand, older people's safety when crossing the street significantly improves in less complex situations, such as in one-way streets (Oxley et al., 1997). Older pedestrians have been observed to be more likely to be hit during the second half of the crossing, i.e. on the far side of the two-way street (Fontaine and Gourlet, 1997; Oxley et al. 1997). A recent experimental study shows that whereas young participants consider the time gaps available in both lanes to decide whether or not to cross the two-way street, older participants make their decisions mainly on the basis of the gap available in the near lane, thus neglecting the far lane (Dommes et al., 2014). Because older pedestrians mainly check traffic approaching in the near lane before starting to cross, they may find that, when reaching the middle of the street, cars approaching from the opposite direction are too close. These 
non-optimal choices could be compensated for by walking faster; but, this is only the case if the pedestrian is able to do so, and if she/he watches for traffic while crossing the street. However, older pedestrians have been shown to pay more attention to watching their step as they cross, causing them to at least partly disregard the approaching traffic (Avineri et al., 2012). At signal-controlled intersections, older pedestrians have even been observed to not look at the traffic at all (Job et al., 1998).

Studies about traffic characteristics also reveal that ageing leads to more frequent unsafe street-crossing decisions when the speed of approaching vehicles is high (Dommes and Cavallo, 2011; Dommes et al., 2014; Lobjois, and Cavallo, 2007, 2009). Because an approaching vehicle is farther away at a high speed than at a low one for a given available time gap, older people more often decide that it is safe to cross, walk more slowly, and choose to cross in shorter safety margins when the speed of the approaching vehicle is high. The use of such distance-based heuristics in older pedestrians (i.e. "the vehicle is far away, I cross" versus "the vehicle is close, I don't cross") is actually related to the misperception of the time available for crossing. Lobjois and Cavallo (2007, 2009), Dommes and Cavallo (2011), and Dommes et al. (2013, 2014) showed different crossing decision patterns in younger pedestrians who appear to select similar average time gaps regardless of speed. In contrast, Oxley et al. (2005) observed that young adults can also make crossing judgements primarily based on vehicle distance but this strategy could be reasonably safe for young adults because they are able to walk fast enough to avoid even relatively close vehicles. In contrast, it clearly leads to risky crossing decisions in slower older participants. The misperception of time-toarrival by older pedestrians has actually been shown to be a significant predictor of unsafe crossings in one-way (Dommes and Cavallo, 2011) and two-way traffic situations (Dommes et al., 2013). 
Studies about pedestrian road crossing behaviour also confirm that advancing age is associated with an increase in risk. However, contrary to research on driving behaviour where empirical evidence is abundant (for a review, see e.g., Anstey et al., 2005), unequivocal empirical evidence for road-crossing behaviour is still scarce. To our knowledge, to date, only two multidimensional and experimental studies have investigated the role of functional decline associated with ageing in street-crossing difficulties: these are by Dommes and Cavallo (2011) and Dommes et al. (2013). That said, the first study only examined decisions in a one-way traffic situation and, as already stated, this is not the situation in which most pedestrian collisions occur. The second study used a judgment task in a simulated two-way traffic environment. However, it did not allow walking speed adaptations to be studied and the selection of an insufficient gap to be compensated for by walking faster. These two studies have, however, specifically highlighted the role of declining visual, cognitive and motor abilities in explaining the greater probability of older pedestrians being involved in a collision whilst crossing the street. Visual processing speed and visual attention abilities, which were assessed using the Useful Field of View test (UFOV ${ }^{\circledR}$ Test, Ball et al., 1993), were common significant predictors, as were executive functions. Whereas several tests have been used to assess visual, perceptual and cognitive functions, pedestrians' motor abilities have only been assessed using the measure of walking speed (Holland and Hill, 2010; Dommes and Cavallo 2011; Dommes et al., 2013). Walking speed decrement has often been mentioned when seeking to explain older people's difficulties in crossing the street probably because it exposes the individuals to potential collisions over a longer period of time. A reduction in walking speed is one of the most obvious and well-known features of age-related decline (see e.g., Prince et al., 1997). However, changes in other gait parameters have also been observed, and a decrease in step length has been shown to have a strong influence on the maintenance of stability whilst walking (Espy et al., 2010). Moreover, recent research has highlighted gait 
variability (step-to-step fluctuations) as a useful and discriminative measure of gait performance compared with routine spatio-temporal measures such as walking speed (see e.g., Lord et al., 2011). The study of such gait parameters could be particularly interesting in situations where pedestrians manage the presence of curbs. This has been little studied in the context of street crossing (Knoblauch et al., 1996; Naveteur et al., 2013), despite the fact that many older pedestrians have reported difficulties in negotiating curbs (Coffin and Morrall, 1995). Obstacle negotiation becomes a challenging task with ageing; indeed, it is among the most commonly reported causes of falls (Campbell et al., 1990).

Thus, there is increased knowledge about the role of some infrastructure or traffic characteristics, as well as factors linked to the ageing process that could explain the fact that older pedestrians are at a greater risk of being involved in a collision. Nonetheless, most of experimental studies have limited the analysis to one-way traffic situations (Oxley et al., 2005; Dommes and Cavallo, 2011; Lobjois and Cavallo, 2007, 2009) and to the decrease of walking speed in studying the role of motor abilities in street-crossing difficulties with ageing (Holland and Hill, 2010, Dommes et al., 2013). A few studies have examined pedestrian behaviours in two-way traffic situations, but these are rare. Furthermore, they have tended to use a judgment task (Dommes et al., 2013) or have asked participants to take only one step forward (Holland and Hill, 2010). A task that allows pedestrians to negotiate an actual twoway street crossing, where curbs are present on both sides of the street (as used in the present study), may put pedestrians in a cognitively and physically difficult situation. To our knowledge, this may more closely match real-life constraints than is the case for existing experimental studies.

Within this framework, the aim of the present study was to gain a better understanding of the risk factors that heighten the probability of older pedestrians being involved in a collision when crossing a two-way street with curbs. In particular, the aim is to investigate the 
effects of age, traffic complexity (one-way vs. two-way traffic situations), time gap availability in each lane, and speed of approaching cars in a safe and controlled simulated environment. A battery of tests was used to assess visual and cognitive abilities, and gait parameters were also recorded during the experiment in order to investigate the impact of agerelated decline on the difficulties of older pedestrians in crossing the street in a cognitively and physically challenging situation.

Based on crash statistics and the existing literature, we hypothesized that older pedestrians would experience more difficulty than younger pedestrians in selecting safe gaps to cross a street, with more decisions that led to collisions with approaching cars as age increases. Age-related difficulties would be more pronounced in two-way traffic situations than in one-way traffic situations, from no difficulties in young pedestrians to greater difficulties in older pedestrians in two-way traffic situations particularly. This expectation is in line with studies by Oxley et al. (1997) and Fontaine and Gourlet (1997). The difficulties experienced by older pedestrians in two-way traffic situations may be particularly more strongly pronounced on the far side of the street (Dommes et al., 2014; Oxley et al., 1997). According to earlier works (Dommes and Cavallo, 2011; Dommes et al., 2013, 2014; Lobjois and Cavallo, 2007, 2009), a significant higher number of unsafe crossings as speed increases should also be observed in older pedestrians only. Globally, these age-related street-crossing difficulties are supposed to be linked to visual and cognitive decrements as well as to a decline in gait parameters. Visual, cognitive and motor deficits are therefore expected to be observed on the performance of participants to the battery of functional tests, with poorer performance as age increases. Performance on these tests are supposed to be associated with unsafe crossing decisions, expressed here as collisions. These associations between functional performance and collisions will be analyzed by the means of a logistic regression analysis. More precisely, gap-selection difficulties are expected to be related to a decline in being able 
to rapidly explore and process visual information in the environment in order to identify a suitable available time gap between approaching cars. Thereafter, a decline in motor abilities may prevent older pedestrians from adjusting their walk accordingly.

\section{Method}

\subsection{Participants}

A total of 78 participants took part in the experiment: 20 young adults ranging in age between 18 and 25 years (M=22.2, SD=1.94), 25 younger-old adults ranging in age between 60 and 72 years (M=67.8, SD=3.35), and 33 older-old adults ranging between 72 and 92 years ( $M=77.2, S D=4.4)$. The younger-old and older-old groups were respectively below and above the mean age (72 years old) of all older participants. The young group of participants and the younger-old group were both comprised of $60 \%$ women and $40 \%$ men, and the older-old group of participants was comprised of $69.7 \%$ women and $30.3 \%$ men.

The criteria for including participants in the younger-old and older-old groups were that they had to be: (i) older than 60 years, (ii) living independently and (iii) commonly getting out and about without any help. These participants were also required to take the Mini-Mental State Examination test (MMSE, Folstein et al., 1975) to ensure the absence of pathological ageing symptoms; only the participants who had a score of above 26 (/30) were included.

The sample was composed of volunteers who answered advertisements distributed around the laboratory with instructions about how to opt in to the study. No reference was made to the examination of age-related difficulties, but to the understanding of street-crossing decision-making processes across the lifespan. The study was approved by the institutional ethics committee. All participants signed an informed consent form before the beginning of the study. 


\subsection{Procedure}

Participants were involved in two sessions. First, they completed a 1.5 -h session to assess their street-crossing behaviours using a simulated road-crossing environment. Approximately one week later, participants answered the battery of visual and cognitive tests.

\subsubsection{The street-crossing simulator}

Street-crossing behaviour was studied using an immersive, interactive street-crossing simulator (see Fig. 1). The device was comprised of a portion of a 5.7-m-wide experimental street on which the participants actually walked. It also included an image-generation system, a ten-screen visual projection system, a 3D sound-rendition system, and a recording system. The projection system provided the pedestrian with a horizontal visual field of between $180^{\circ}$ (at the starting point of the crossing) and $300^{\circ}$ (in the middle of the street and at the pavement on the other side), and a vertical visual field of $40^{\circ}$. The images (60 frames per second) were calculated and projected at each participant's eye height. Scenes were updated interactively by a movement-tracking system (Vicon ${ }^{\circledR}$, Oxford Metrics, London, UK) that also recorded each participant's locomotion (sub-millimetre accuracy) using eight reflective markers placed on the head of each participant. Five other retro-reflective spherical markers were attached to each of the participant's feet (between the lateral malleoli and the heel) to record gait parameters throughout the street-crossing task (at a sample rate of $100 \mathrm{~Hz}$ ). The visual scenes represented a two-way street of $5.70 \mathrm{~m}$ in width from pavement to pavement $(15.4 \mathrm{~cm}$ high). The traffic consisted of groups of motorcycles and cars (between 5 and 10 vehicles) moving at a constant speed. The direction of the traffic followed the French traffic rules: in the nearside lane (2.85 m wide), the flow of vehicles approached from the left of the pedestrian, who was standing on the pavement. Vehicles in the far lane (2.85 m wide) approached from the right. The pedestrian's initial position was such that she/he could watch the traffic coming from both directions by turning her/his head to the left or right. Two wooden curbs $(15.4 \mathrm{~cm}$ 
high, see Fig. 1) were positioned on both sides of the experimental street and participants wore a safety harness to prevent them from falling.

\section{[FIGURE 1]}

\subsubsection{The street-crossing task}

Participants were tested individually on the simulator. The experimenter began the session by describing the basic principles of the task and allowed participants walking without approaching traffic in the simulated two-way street, stepping on and off the curbs and becoming used to the safety harness. Then the participant performed a maximum of 18 practice trials with approaching cars from one and two directions. Once the participants were comfortable and fully understood the task, they performed the experimental street-crossing task, which was composed of 2 blocks of 21 trials, with a break between the blocks. The street-crossing experimental session (recording session) took about 30 min to complete. No participants reported suffering from simulator sickness symptoms, maybe because the task involved an active walk of more than $7 \mathrm{~m}$.

For each trial, participants had to judge whether the available gaps in the approaching traffic were suitable for crossing the street. They were instructed to choose traffic gaps in which they could cross the entire two-way street without running and/or stopping in the middle of the street. If they thought it was safe to do so, they were instructed to cross. Otherwise, they waited for the next trial. The participants' decision to cross or not cross, and their motion until they reached the opposite pavement, was recorded.

Across the 42 trials, traffic complexity (vehicles approaching from one or two directions), lane (near versus far side of the street), and vehicle speed (40 or $60 \mathrm{~km} / \mathrm{h}$ ) were varied: 10 trials involved vehicles that approached from the near lane only, at $40 \mathrm{~km} / \mathrm{h}$ (5 trials) and at $60 \mathrm{~km} / \mathrm{h}$ (5 trials); 10 trials involved vehicles that approached from the far lane only, at $40 \mathrm{~km} / \mathrm{h}$ (5 trials) and at $60 \mathrm{~km} / \mathrm{h}$ (5 trials); and 22 trials involved vehicles that 
approached from both directions of the two-way street, at $40 \mathrm{~km} / \mathrm{h}$ (11 trials) and at $60 \mathrm{~km} / \mathrm{h}$ (11 trials). Across trials, the available time gap(s) between two approaching cars in the traffic flow(s) was (were) varied (see Fig. 1), but in such a way that all pedestrians had the same number of opportunities to cross the street, as a function of their own walking speed (WS). Before the beginning of the street-crossing task, WS over a distance equivalent to the width of the simulated street was measured in the simulator room (but with no approaching virtual cars) on six trials at a normal-to-fast walking pace. Each individual's WS (the average of the six trials) was then entered into the street-crossing scenario and used for the entire experiment. During the street-crossing task, participants were instructed to walk at a normalto-fast speed, but they were also advised that they could walk faster or slower if they wanted to. Rather than proposing fixed gaps, and to ensure that all participants were presented with gaps that yielded comparable actions, the temporal values of the gaps proposed were modified, based on each individual's WS. This modification could be $-50 \%,+50 \%,+100 \%$ or $+150 \%$ of each individual's mean WS, making a total of 5 possible time gaps per lane and per participant (WS; WS-50\% of WS; WS+50\% of WS; WS+100\% of WS; WS+150\% of WS). In the case of a negative modification, for example, the available gap was shorter than the individual's WS, while in the case of a positive modification, the gap was longer than the individual's $\mathrm{WS}^{1}$. In one-way traffic situations, the five possible time gaps were all proposed in each lane, and for each of the two speeds of approaching cars (40 and $60 \mathrm{~km} / \mathrm{h}$ ), making a total of 20 trials randomly presenting vehicles approaching from one direction. When traffic was approaching from both directions, gaps could be the same in each lane (5 possible time gaps for each speed of approaching cars, for a total of 10 trials), or different in each lane to

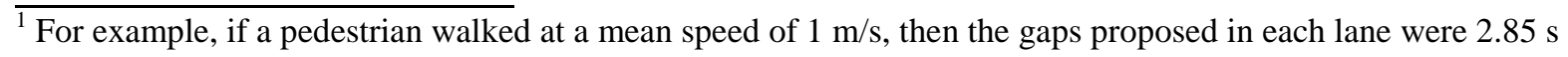
(WS); $1.43 \mathrm{~s}$ (WS - 50\% of WS); $4.28 \mathrm{~s}$ (WS + 50\% of WS); $5.70 \mathrm{~s}$ (WS + 100\% of WS); and $7.13 \mathrm{~s}$ (WS + $150 \%$ of WS).
} 
allow situations where time gaps were shorter in the near lane (3 trials for each speed, for a total of 6 trials) or shorter in the far lane (3 trials for each speed, for a total of 6 trials). In the 22 two-way traffic situations, varying time gaps were always synchronized and, thus, were simultaneously available in both lanes (bearing in mind that time gap durations could however be different in each lane for some trials). The manipulated gaps always appeared $6 \mathrm{~s}$ after the onset of the trial, providing a view of the scenes for $6 \mathrm{~s}$ before participants could cross. All other vehicles in the traffic groups were separated by about $1.5 \mathrm{~s}$ so that participants could not cross between them. The 42 trials were randomly presented across 2 blocks of 21 trials.

The behavioural indicator used for the present study was the percentage of collisions. A crossing was scored as a collision when the participant was 'hit' by the approaching car, i.e. she/he was in front of the approaching car (somewhere between the right and left sides of the car) when it passed the crossing line. This variable was calculated for the near and far lanes of the two-way street and was expressed as a percentage of the total number of crossings made by the participant.

\subsubsection{The assessment of visual, cognitive and motor abilities}

In addition to the street-crossing task, all participants took a battery of visual and cognitive performance tests, which was comprised of one visual test (i.e. binocular far acuity), and six cognitive tests, i.e. the three $\mathrm{UFOV}^{\circledR}$ subtests and the shifting, inhibiting and updating tasks. These tests were chosen in line with previous research on pedestrians' behaviour (Dommes and Cavallo, 2011, Dommes et al., 2013). These tests are also the most famous ones to show age-related decrements and significant associations with driving difficulties (Anstey et al., 2005). They took approximately one hour to complete. Gait parameters were recorded during the street-crossing experimental session (see also section 2.2.2). 
Visual test. Each participant completed an eye examination using a subtest of Ergovision ${ }^{\circledR}$ (Essilor). This standard device for eye disease screenings allowed us to assess the binocular acuity for far vision. In this subtest, the participants had to recognize letters and numbers of the smallest possible height. The far vision score (out of 12 points) was considered.

\section{Cognitive tests:}

UFOV $^{\circledR}$ test. Processing speed and visual attention were assessed using the UFOV ${ }^{\circledR}$ test (Ball, et al., 1993), which is a computer-based test of rapid visual-scene perception, without eye or head movements. Three subtests measured the individual's speed of processing and visualattention abilities across increasingly complex visual displays, i.e. central target identification alone (Subtest 1), central target identification with peripheral target localization (Subtest 2), and central target identification with peripheral target localization in the presence of distractors (Subtest 3). In each subtest, $\mathrm{UFOV}^{\circledR}$ automatically adjusted the stimulus-display time in milliseconds, as needed: after two correct responses, stimulus display time for the next item was shortened; it was lengthened if a response was incorrect. This process of tracking the perceptual thresholds (ms) continued until a stable estimate of $75 \%$ correct was obtained for each subtest. For all subtests, stimulus durations ranged between 17 and 500 ms.

Shifting task. Shifting was assessed using a subtest of the TAP ${ }^{\circledR}$ (Tests of Attentional Performance $^{\circledR}$, a computer-based task (Zimmerman and Fimm, 2010). The procedure was a "set shifting" task, in the same vein as the famous Trail Making Test (Reitan, 1958). Either angular or round figures were simultaneously presented to the right and left of the centre of the monitor. In the first subtest, the participant was required to react only to the angular shapes (50 targets), by pressing the left or right key according to whether the target stimulus (the angular shape) appeared to the left or the right of the centre of the monitor. In the second subtest, the participant was required to react only to the round shapes (50 targets), by pressing the left or right key according to whether the target stimulus (the round shape) appeared to the 
left or the right of the centre of the monitor. The third subtest was composed of 100 trials and the type of target stimulus was alternated across trials (angular - round - angular - round, etc.). Cues were given on the first trial and whenever the participant responded incorrectly. The difference between the median reaction times on subtests 1 and 2 and subtest 3 was used to describe a shifting cost.

Inhibiting task. Inhibition abilities were also measured via a subtest of the $\mathrm{TAP}^{\circledR}$. In line with the Simon (Craft and Simon, 1970) and spatial Stroop (Logan and Zbrodoff, 1979) paradigms, a left-pointing or right-pointing arrow target was displayed randomly on the left, in the centre, or on the right of the computer screen (a total of 60 times). Participants were required to determine the direction of the arrow while ignoring its location. There were 30 compatible targets (e.g., a left-pointing arrow presented on the left side of the screen), and 30 incompatible targets (e.g., a right-pointing arrow presented on the left side of the screen). The difference between the median reaction times to identify the compatible and incompatible stimuli was used to describe an inhibiting cost.

Updating task. The control of information flow and updating of information in working memory were assessed using the working memory subtest of the $\mathrm{TAP}^{\circledR}$. Numbers were displayed one at a time on a computer screen. For each of the 100 trials presented (15 of which were targets), participants were required to press a response button when any number was the same as the number displayed two numbers earlier. For example, the sequence 3728 5 required no response, but the sequence 37828 required a response because of the two 8's. The number of correct answers was used in the present study to reveal a failure to control information flow.

Motor test: Two gait parameters were recorded each time the participant attempted to cross the street in the experimental task. The mean step length $(\mathrm{cm})$ was computed as the horizontal distance between the retro-reflective spherical markers attached to the feet of the participant. 
The first and last steps interacting with the curbs were excluded from this calculation. A ratio between the height of the participant and her/his step length was considered in the present data analyses. Indeed, the participants' height was significantly different between age groups, $\mathrm{F}(2,75)=3.16, \mathrm{p}<.05, \eta^{2}=.08$. Older-old participants were significantly smaller $(\mathrm{M}=162.24 \mathrm{~cm}$, $\mathrm{SD}=9.23)$ than younger-old $(\mathrm{M}=167.88 \mathrm{~cm}, \mathrm{SD}=11)$ and young $(\mathrm{M}=167.5 \mathrm{~cm}, \mathrm{SD}=7.77)$ participants $(\mathrm{p}<.05)$. As step length depends on the individual's height, a ratio was calculated (length(cm)/height(cm)). The higher the ratio, the larger are the steps. Step length variability (in \%, SD/Mean x 100) was also calculated.

\section{Results}

\subsection{Data analysis}

Analyses of variance (ANOVAs) were conducted to examine all dependent measures obtained from the street-crossing task and the functional tests. Statistical significance was set at a p value of 0.05 . The computation of relative effect size $\left(\eta^{2}\right)$ and post-hoc comparisons (LSD Fisher) completed the analyses. A logistic regression analysis was then computed to identify the predictors of the decisions that led to collisions.

\subsection{Street-crossing task}

The 3 (age group: young, younger-old and older-old participants) x 2 (traffic complexity: one-way and two-way traffic situations) x 2 (lane: near and far lanes) x 2 (speed: 40 and $60 \mathrm{~km} / \mathrm{h}$ ) ANOVA revealed a significant main effect of age group on collisions, $F(2,75)=4.34, p<.05, \eta^{2}=.10$, see Figure 2. Post-hoc tests indicated that older-old participants made significantly more decisions that led to collisions with approaching cars than both groups of younger-old and young participants $(\mathrm{p}<.05)$. The difference between younger-old and young participants was not significant $(\mathrm{p}=.35)$.

[FIGURE 2] 
The results also indicated a significant main effect of traffic, $F(1,75)=23.40, p<.01$ $\eta^{2}=.24$, with a higher percentage of collisions in two-way traffic situations ( $M=4.05 \%$, $\mathrm{SD}=7.35)$ than in one-way traffic situations $(\mathrm{M}=1.32 \%, \mathrm{SD}=4.01)$.

The main effect of lane was also significant, $F(1,75)=26.43, p<.01 \eta^{2}=.26$, with a higher percentage of collisions in the far lane $(M=4.56 \%, S D=8.13)$ than in the near lane $(M=0.81 \%$, $\mathrm{SD}=3.22)$

The main effect of speed was also significant, $F(1,75)=27.8, \mathrm{p}<.01 \eta^{2}=.27$, with a higher percentage of collisions at $60 \mathrm{~km} / \mathrm{h}(\mathrm{M}=4.41 \%, \mathrm{SD}=7.72)$ than at $40 \mathrm{~km} / \mathrm{h}(\mathrm{M}=0.96 \%$, $\mathrm{SD}=3.6)$

Contrary to our expectations, the interaction between age group and traffic complexity was not significant $(\mathrm{F}(2,75)=0.48, \mathrm{p}=.62)$. The three groups of participants made significantly more decisions that led to collisions when the traffic was coming from two directions than from one direction (cf. Fig. 3a), and no significant group-related differences were apparent.

The interaction between age group and lane was significant, $F(2,75)=3.6, p<.05, \eta^{2}=.09$. Post-hoc tests revealed that the both groups of older participants made significantly more decisions that led to collisions in the far lane than in the near lane $(\mathrm{p}<.05)$, whereas lane differences were not significant in the young group of participants (see Fig. 3b). Furthermore, while differences between the three age groups were not significant in the near lane, in the far lane, older-old participants made more decisions that led to collisions than young participants and younger-old participants $(\mathrm{p}<.01)$.

The results also indicated a significant interaction between age group and speed, $\mathrm{F}(2,75)=3.20, \mathrm{p}<.05, \eta^{2}=.08$. While speed differences were not significant in the young group, post-hoc tests indicated that younger-old and older-old groups made significantly $(\mathrm{p}<.05)$ more decisions that led to collisions when vehicles were approaching at $60 \mathrm{~km} / \mathrm{h}$ than at 40km/h (see Fig.3c). At 40km/h, group differences were not significant. On the contrary, at 
$60 \mathrm{~km} / \mathrm{h}$, older-old participants made more decisions that led to collisions than the younger-old group and the young group of participants $(\mathrm{p}<.01)$.

\section{[FIGURE 3]}

The interaction between traffic and lane was significant, $F(1,75)=19.6, p<.01, \eta^{2}=.21$, as was the interaction between traffic and speed, $\mathrm{F}(1,75)=12.4, \mathrm{p}<.01 \eta^{2}=.14$, lane and speed, $\mathrm{F}(1,75)=11.9, \mathrm{p}<.01 \eta^{2}=.14$, and traffic, lane and speed $\mathrm{F}(1,75)=7.41, \mathrm{p}<.01 \eta^{2}=.09$. These last interactions are not detailed here because our study was particularly focused on age differences.

The interaction between age group $x$ lane $x$ speed was significant, $F(2,75)=3.66, p<.05$ $\eta^{2}=.09$. In the near lane, the three groups of participants showed similar tendencies, with no significant speed-related differences. On the contrary, in the far lane, only the older-old group of participants shows significant speed-related differences.

The interactions between age group x traffic x lane, between age group x traffic x speed, and between age group $\mathrm{x}$ traffic $\mathrm{x}$ lane $\mathrm{x}$ speed were not significant.

\subsection{Visual and cognitive abilities}

Performances on the visual and cognitive tests are presented in Table 1.

\section{[TABLE 1]}

A one-way ANOVA revealed a significant effect of age group on visual acuity, $\mathrm{F}(2,75)=8.25, \mathrm{p}<.01, \eta^{2}=.18$. Older-old participants had a lower mean score than the youngerold $(\mathrm{p}<.05)$ and young participants $(\mathrm{p}<.01)$. The difference between younger-old and young participants was close to the level of significance $(p=.054)$.

Results showed a significant effect of age group on speed of processing scores obtained for the UFOV ${ }^{\circledR}$ Sub-test $1, F(2,75)=4.67, \mathrm{p}<.05, \eta^{2}=.11$, with older-old participants having higher threshold scores than younger-old and young participants (p’s<.05). The difference between the younger-old and young groups was not significant. 
The effect of age group was also significant on the measure of divided attention collected from the UFOV® Sub-test $2, \mathrm{~F}(2,75)=16.54, \mathrm{p}<.01, \eta^{2}=.31$, with older-old participants having higher threshold scores than younger-old and young participants (p’s<.01). The difference between younger-old and young participants was significant $(\mathrm{p}<.01)$.

Age group differences were again significant on the measure of selective attention collected from the UFOV® Sub-test $3, \mathrm{~F}(2,75)=52.18, \mathrm{p}<.01, \eta^{2}=.58$, with older-old participants having higher threshold scores than the two other groups (p’s<.01). The difference between younger-old and young participants was also significant $(\mathrm{p}<.01)$.

Age group differences were also significant on shifting performance, $F(2,75)=11.61$, $\mathrm{p}<.01, \eta^{2}=.24$, with older-old and younger-old participants having higher shifting costs than young participants (p’s<.01). The difference between the two older groups was not significant.

Age group differences were furthermore significant on inhibiting performance, $\mathrm{F}(2,75)=13.58, \mathrm{p}<.01, \eta^{2}=.27$, with older-old and younger-old participants having higher inhibiting costs than young participants (p's<.01). The difference between the two older groups was also significant $(\mathrm{p}<.05)$.

Finally, age group differences were significant on updating performance, $\mathrm{F}(2,75)=25.36, \mathrm{p}<.01, \eta^{2}=.40$, with older-old and younger-old participants having significantly fewer correct answers than young participants $(\mathrm{p}<.01)$. The difference between the two older groups was significant $(\mathrm{p}<.01)$.

\subsection{Gait parameters while crossing the street}

One-way ANOVAs showed significant group differences for both measures of gait parameters, i.e., step length ratio $\mathrm{F}(2,75)=25.83, \mathrm{p}<.01, \eta^{2}=.41$ and step length variability $\mathrm{F}(2,75)=3.08, \mathrm{p}=.052, \eta^{2}=.08$. Post-hoc tests indicated that the steps taken by the two older 
groups of participants were significantly shorter than those of the young group of participants ( $<<.01$, see Table 2). The difference between younger-old and older-old was not significant. Post-hoc tests also revealed that the step length of both groups of older participants was slightly more variable than the step length of the young participants $(\mathrm{p}<.05$, see Table 2$)$. There were no significant differences between the two older groups $(p=.74)$.

[TABLE 2]

\subsection{The role of visual, cognitive and gait characteristics in the safety of street-crossing decisions}

To examine the role of visual, cognitive, and gait parameters in the decisions that led to collisions, a logistic regression analysis was conducted. The binary outcome to be predicted took the value " 1 " for a decision that led to a collision, and " 0 " for a decision that did not lead to a collision. In total, 3276 decisions (42 decisions x 78 participants) were considered. Binary logistic regression is a useful method to model the event probability for a categorical response variable with two outcomes (e.g. no collision/collision). Several statistics were used to interpret the results. The first three were to test: (i) the significance of the model itself (Chi square with a p value of less than 0.05 ); (ii) the variability in the dependent variable that could be explained by the model (Nagelkerke's pseudo r-squared ranged from 0 to 1); and (iii) whether the model adequately describes the data (Hosmer-Lemeshow goodness-of-fit statistic with a p value of more than 0.05 ). An odds ratio (OR) was then used to determine the probability that the categorical response outcome variable would occur given a particular exposure of a predictive factor (range between 0 and infinity). Here, an OR that is strictly equal to 1 means that exposure does not affect odds of the outcome, an OR $>1$ means that exposure is associated with higher odds of the outcome, and an $\mathrm{OR}<1$ means that exposure is associated with lower odds of the outcome (Szumilas, 2010). The 95\% confidence interval (CI) is used to estimate the precision of the OR. A large CI indicates a low level of precision 
of the OR, whereas a small CI indicates a higher precision of the OR. CI is often also used as a proxy for the presence of statistical significance if it does not cross the null value (i.e., OR=1, Szumilas, 2010).

Predictors of collisions were entered as follows in the hierarchical logistic regression analysis: (i) performance on the visual and cognitive tests were entered in a first step to illustrate the abilities involved in the decision-making process of gap-selection decisions; (ii) gait parameters were entered in a second step to illustrate the motor abilities involved in crossing the street and potentially adjusting gait to match the perception of incoming traffic; and (iii) chronological age was entered in a final step (step 3) to determine if age predicted the occurrence of a collision after differences in visual, cognitive and motor abilities were taken into account.

Results are presented in Table 3.

The final model was significant $\chi 2(10)=35.24, \mathrm{p}<.001$. When visual and cognitive abilities were included in the model (step 1), visual acuity, speed of processing and selective attention emerged as significant predictors. The probability of a pedestrian taking a decision that led to a collision was significantly associated with: (i) poorer visual acuity, (ii) slower processing speed, and (iii) decline in selective attention. The inclusion of gait parameters in the second step was significant. Step length emerged as the only one significant predictor. Selective attention was no more significant, but visual acuity and speed of processing were still significant predictors. The probability of a pedestrian experiencing a collision with an approaching car while crossing the street was therefore significantly associated with: (i) poorer visual acuity, (ii) slower processing speed, and (iii) shorter step length. Finally, the forced inclusion of age in the final step did not account for a statistically significant amount of additional predictions of collision occurrence.

[TABLE 3] 


\section{Discussion}

The aim of the present study was to gain a better understanding of the risk factors that heighten the probability of older pedestrians being involved in a collision when crossing a two-way street with curbs. In line with previous research (Oxley et al. 2005; Lobjois and Cavallo 2007, 2009; Holland and Hill 2010), results showed that ageing does lead to an increased risk of collisions. Older-old participants (over 72 years) made more decisions that led to collisions with approaching vehicles than younger-old participants (60 to 72 years) and young participants (18 to 25 years). But younger-old participants did not make significantly more dangerous decisions than young participants. These results are in line with French accident statistics that indicate a higher incidence of pedestrian accidents at a very old age only ( $>75$ years). Although people aged over 75 years represented 37.2\% of pedestrian fatalities in 2011 in France, pedestrians aged between 65 and 74 years, and between 18 and 24 years represented only $11.8 \%$ and $8.5 \%$ of pedestrian fatalities, respectively (ONISR, 2012).

The study of causal factors related to traffic did not reveal complexity as a particularly significant difficulty for this group of participants. Indeed, contrary to our hypothesis, twoway street crossing was difficult for all three groups of participants, and not just for the olderold ones. Whilst the young-old and older-old participants were found to experience difficulties in managing two-way traffic situations, confirming some previous observational studies (e.g., Oxley et al., 1997), so too did the young participants. Processing the visual information available in both lanes of a two-way street may involve high attentional demands for both young and older individuals. These results are yet to be confirmed by further observational and experimental studies.

With advancing age, it seems to be particularly difficult to consider the second lane in which traffic can also approach in a two-way traffic situation. Contrary to young participants, both groups of older participants had more collisions in the far lane than in the near lane. This 
result is in line with the few published papers on this matter, i.e. the observational study by Oxley et al. (1997), the accident analysis by Fontaine and Gourlet (1997) and the recent experimental study by Dommes et al. (2014). Biased decision-making that favours the near lane may reveal that older pedestrians adopt inadequate visual exploration strategies. There may also be a substantial cognitive overload when faced with a demanding situation such as a two-way street crossing.

Results also highlighted that ageing leads to difficulties in taking into account the speed of approaching vehicles in the decision-making process. Contrary to young participants, the two older groups had more collisions when vehicles were approaching at $60 \mathrm{~km} / \mathrm{h}$ than at 40km/h. This finding is in line with previous studies (Dommes et al., 2014; Lobjois and Cavallo, 2007, 2009), which have indicated that older pedestrians have difficulty in processing the speed of approaching cars. Perceptual decline associated with normal ageing may actually limit people’s perception of moving objects (Snowden and Kavanagh, 2006). As a consequence, older participants base their decisions to cross the street on the distance of approaching vehicles. When high speeds are involved, this constitutes very dangerous behaviour.

To understand why older pedestrians particularly encounter difficulties when crossing the street, we used a battery of tests to assess participants’ visual and cognitive abilities. Gait parameters were also recorded whilst participants were crossing the street. The significant age-related decrements in visual and cognitive abilities observed in the present experiment are in line with those widely reported in the literature of ageing. Normal ageing is commonly associated with the decline of several visual functions such as acuity (for a review, see Owsley, 2011; Owsley and McGwin, 2010). Cognitive functions also decline from the age of 65 (e.g., Schaie, 1989, for a review, see also Lemaire and Bherer, 2005), both in terms of the speed of processing visual information (e.g., Salthouse, 1996) and in controlling attention 
(e.g., Verhaeghen and Cerella, 2002). A decline in executive functions (e.g., Salthouse et al., 2003) is particularly marked in complex situations, and the street-crossing task appears to be one such situation.

Significant age-related differences in gait parameters were also observed. These illustrate a well-known decrease in motor abilities associated with normal ageing (e.g., Spirduso et al., 2005). The length of the steps taken by the two groups of older participants in the present experiment was observed to decrease, whereas their step length variability was observed to increase. These results may indicate a reorganization of the gait pattern with ageing to avoid falls and stumbles. With normal ageing, the degeneration of one or more of sensory systems occurs and may compromise balance and walking (for a review, see e.g., Prince et al., 1997). To keep their balance and maintain a safe gait (i.e. without falls), older people have been shown to involve more attentional resources than young adults (Beauchet and Berrut, 2006). Sensorimotor performance seems to become cognitively more demanding with advancing age (e.g., Lindenberger et al., 2000; Woollacott, and Shumway-Cook, 2002). With ageing, gait performance cannot be conceived as a series of identical and automatic steps. Instead, gait becomes a complex task that places demands on sensory and cognitive systems (Sheridan and Hausdorff, 2007). Given their fear of falling (e.g., Scheffer et al., 2008) and their need to keep their balance when walking (Woollacott and Tang, 1997), older pedestrians seem to allocate more attention to watching their steps as they cross, causing them to at least partly disregard the approaching traffic (Avineri et al., 2012). The presence of curbs at the beginning and at the end of the present street-crossing task may add supplementary cognitive and motor demands that very few studies have precisely examined. Recently, Naveteur et al. (2013) observed that curbs led to longer crossing durations, and that this effect tended to increase with ageing and with the fear of falling. As compared to younger adults, the supplementary time for older pedestrians to step on and off the curbs may reveal motor 
difficulties with ageing that affect their time for crossing, but this may also reveal greater attentional demands devoted to the current task (i.e. avoid falling and control balance). This supplementary attentional demands (as compared to the absence of curbs) may have consequences on the available cognitive resources devoted to watch the approaching traffic. Such consequences warrant further investigation.

Regression analyses showed that visual acuity may play an important role in explaining gap-acceptance difficulties with ageing. Crossing the street is a highly visual task (Shinar and Scheiber, 1991); thus, the decline in visual acuity experienced by older adults (see e.g., Haegerstrom-Portnoy et al., 1999) may be an important factor in explaining their greater likelihood of making unsafe decisions when crossing a two-way street.

Processing-speed and visual-attention abilities assessed through the $\mathrm{UFOV}^{\circledR}$ test were also shown to explain the increased likelihood for older pedestrians to be involved in collisions. Ageing may lead to difficulties in rapidly exploring and processing visual information from the environment, thus impairing the street-crossing decision-making process followed by older pedestrians. In turn, this may lead them to wrongly identify an available time gap. They may also neglect to include speed information and traffic approaching in the far lane in their decision-making process. In line with previous studies (Dommes and Cavallo, 2011; Dommes et al., 2013), the score from the $\mathrm{UFOV}^{\circledR}$ test has proven to be a sensitive measure by which street-crossing collisions can be predicted. Visual attention has indeed been shown to play an important role in predicting difficulties among older drivers (Ball and Owsley, 1991; Ball et al. 1993), mobility decrements and falls among older walkers (Owsley and McGwin, 2004). The increased likelihood of collisions involving those diagnosed with mild dementia also seems to be associated with impairments in processing speed and visualattention abilities, as assessed by the Useful Field of View test (Dommes et al., in press). 
In the main, results of the present study show that these non-optimal and dangerous street-crossing choices could not be compensated for by the modification of gait parameters, such as speeding up or increasing step length. The regression analysis showed that step length, in particular, was a significant predictor of the occurrence of collisions after visual and cognitive abilities were taken into account. A decrease in step length with ageing may indicate a postural strategy for older pedestrians to improve stability (Bhatt et al., 2005). A decrease in step length is also related to a slowing down in walking speed (Espy et al., 2010). Older people tend to walk more slowly; this is widely perceived as the reason for them being involved in collisions more often, and for their lack of ability to complete a road crossing before new traffic arrives or before traffic signals change (Hoxie and Rubenstein, 1994; Zegeer et al., 1993 cited by Dunbar, 2012). However, the higher incidence of collisions among older pedestrians cannot be understood solely in terms of slower walking speed; rather, it can be ascribed to an incorrect gap acceptance choice and an inadequate subsequent action. This study is actually the first to offer a control for walking speed decrements with ageing. It has done so by experimentally proposing to all pedestrians the same number of opportunities to cross the street as a function of their own walking speed. Even in this context, older pedestrians were observed to make more decisions that led to collisions, indicating that they had specific difficulties in choosing a safe gap to cross and then in adjusting their walking gait accordingly to match their perception of oncoming traffic. Whilst we are still unsure as to whether the older participants were looking at approaching cars while crossing the street, it is certainly known that older pedestrians allocate more attention to watching their step as they cross (Avineri et al., 2012). Even if they were watching traffic while crossing, their non-optimal choices could not be compensated for by walking faster, increasing step length or running because of the decline in their physical abilities (see e.g., Salzman, 2010). When required to change from walking at their preferred speed to walking quickly, older 
people have been shown to fail to achieve the same increases in speed and stride length relative to the increases achieved by young adults (Shkuratova et al., 2004). Running is also dangerous because of the increased risk to fall.

While simulated road environment may not give a correct assessment of crash frequency, the present findings have enhanced our understanding of the visual, cognitive and motor predictors of unsafe street-crossing decisions. In the present study, unsafe decisions were illustrated by the measure of collisions that may not necessarily have resulted in crashes in real-life situations because approaching cars may have taken evasive action. But collisions collected here well reveal incorrect or altered decision-making processes with ageing (in choosing to cross in very short gaps), and impossible or unperceived evasive actions (participants actually walked in the simulator and could therefore run if they were able to do so and if they watched traffic while crossing). If it could be very useful that further investigations study the links between visual, cognitive and motor declines with ageing and crash frequency in a real on-road task, this is quite impossible. One promising issue could be to combine observational data, collected as precisely as possible (with video cameras for example) on all stages of the pedestrians' crossing, and functional data from the same pedestrians (collected through on-site questionnaires and further testing in a laboratory setting).

\section{Conclusion}

Overall, the present study provided a multidimensional explanation of the relationship between ageing and difficulties in safely crossing a two-way street with curbs. It included a combination of declines in visual, cognitive, and motor performance with advancing age. The findings suggest that age per se contributes very little to the prediction of collisions, once the pedestrian's visual, cognitive, and motor abilities are taken into account. The greater gapselection difficulties with ageing seem to be the consequences of altered decision-making 
processes (because of age-related visual and cognitive deficits). Difficulties in motor abilities may then prevent older pedestrians from calibrating action to perception and adjusting their walking pace to compensate for dangerous street-crossing choices. This last difficulty has to be further studied to assess the priority given by participants to the task when faced with the choice between avoiding approaching vehicles or keeping their balance.

Finally, the results of this study have implications for making several recommendations to improve older pedestrians' safety, both in terms of road design and training programmes. Car-free islands in the middle of two-way streets could efficiently reduce the difficulties experienced by older pedestrians when trying to take into account traffic in the far lane during street-crossing decision-making. Car speed reductions could also limit the consequences of their difficulties in judging the speed of approaching cars when crossing the street. Moreover, wider pavements and narrower lanes are known to be efficient countermeasures to increase visibility by removing parked vehicles and reducing the time spent by pedestrians on the road (Ewing, 1999; Zegeer et al. 2002). Furthermore, lowering curb heights is a good engineering countermeasure for older pedestrians in particular. Indeed, it could be widely implemented in urban cities, and not only on marked crossings (i.e. those equipped with zebra and/or pedestrian and traffic lights). Finally, it may be worth exploring how to directly modify the behaviour of older pedestrians (Dommes and Cavallo, 2012; Dommes et al., 2012). For example, a mixed perceptual, cognitive and motor training programme may be able to improve the functional abilities of older people. In turn, this could enhance the selection of safe gaps in which to cross the street and increase the mobility of people on the road. 


\section{REFERENCES}

Anstey, K..J., Wood, J., Lord, S., Walker, J.G., 2005. Cognitive, sensory and physical factor enabling driving safety in older adults. Clinical Psychology Review; 25, 45-65.

Avineri, E., Shinar, D., Susilo, Y., 2012. Pedestrians' behaviour in cross walks: The effects of fear of falling and age. Accident Analysis and Prevention, 44(1), 30-34.

Ball, K.., Owsley, C., 1991. Identifying Correlates of Accident Involvement for the Older Driver. Human Factors, 33, 583-595.

Ball, K., Owsley, C., Sloane, M.E., Roenker, D.L., Bruni, J.R., 1993. Visual Attention Problems as a Predictor of Vehicle Crashes among Older Drivers. Investigative Ophthalmology \& Visual Science, 34, 3110-3123.

Bhatt, T., Wening, J.D., Pai Y.-C., 2005. Influence of gait speed on stability: Recover from anterior slips and compensatory stepping. Gait and Posture, 21, 146-156.

Beauchet, O., Berrut, G., 2006. Gait and dual-task: definition, interest, and perspectives in the elderly. Psychologie \& NeuroPsychiatr ie duVieillissement, 4 (3), 215-25.

Campbell, A.J., Borrie, M.J., Spears, G.F., Jackson, S.L., Brown, J.S., Fitzgerald, J.L., 1990. Circumstances and consequences of falls experienced by a community population 70 years and over during a prospective study. Age Ageing, 19, 136-41.

Coffin, A., Morrall, J., 1995. Walking speeds of elderly pedestrians at crosswalks. Transportation Research Record, 1487, 63-67.

Craft, J.L., Simon, J.R., 1970. Processing symbolic information from a visual display: interference from an irrevelant directional cue. Journal of Experimental Psychology, 3, 415-420

Dommes, A., Cavallo, V., 2011. The role of perceptual, cognitive, and motor abilities in street-crossing decisions of young and older pedestrians. Ophthalmic and Physiological Optics, 31, 292-301. 
Dommes, A., Cavallo, V., 2012. Can simulator-based training improve street-crossing safety for older pedestrians? Transportation Research Part F: Traffic Psychology and Behaviour, 15, 206-218.

Dommes, A., Cavallo, V., Dubuisson, J.B., Tournier, I., Vienne, F., 2014. Crossing a two-way street: comparison of young and old pedestrians. Journal of Safety Research, 50, 27-34.

Dommes, A., Cavallo, V., Oxley, J.A., 2013. Functional declines as predictors of risky streetcrossing decisions in older pedestrians. Accident Analysis and Prevention, 59, 135-143.

Dommes, A., Cavallo, V., Vienne, F., Aillerie, I., 2012. Age-related differences in streetcrossing safety before and after training of older pedestrians. Accident Analysis and Prevention, 44, 42-47.

Dommes, A., Wu, Y.H., Aquino, J.P., Pitti-Ferrandi, H., Soleille, M., Martineau-Fleury, S., Samson, M., Rigaud, A.-S., in press. Is mild dementia related to unsafe street-crossing decisions? Alzheimer Disease and Associate Disorders [Epub ahead of print]

Dunbar, G., 2012. The relative risk of nearside accidents is high for the youngest and oldest pedestrians. Accident Analysis and Prevention, 45, 517-521.

Espy, D.D., Yang, F., Bhatt, T., Pai, Y.C., 2010. Independent influence of gait speed and step length on stability and fall risk. Gait and Posture, 32, 378-382.

Ewing, R., 1999. Traffic calming: state of the practice. Washington, Institute of Transportation Engineers, Federal Highway Administration. http://www.ite.org/traffic/tcstate.asp

Folstein, M.F., Folstein, S.E., McHugh, P.R., 1975. Mini-mental state: A practical method for grading the cognitive state of patients for the clinician. Journal of Psychiatric Research, 12 (3), 189-98.

Fontaine, H., Gourlet, Y., 1997. Fatal pedestrian accident in France: a typological analysis. Accident Analysis and Prevention, 29, 303-312. 
Haegerstrom-Portnoy, G., Schneck, M.E., Brabyn, J.A., 1999. Seeing into old age: vision function beyond acuity. Optometry and Vision Science 76, 141-158.

Holland C, Hill R., 2010. Gender differences in factors predicting unsafe crossing decision in adult pedestrians across the lifespan: a simulation study. Accident Analysis and Prevention, 42,1097-1106.

Job, R.F.S., Haynes, J., Prabhakar, T., Lee, S.H.V., Quach, J., 1998. Pedestrians at traffic light controlled intersections: Crossing behaviour in the elderly and nonelderly. In K. Smith \& B.G. Aitken \& R.H. Grzebieta (Eds.), Proceedings of the Conference on Pedestrian Safety (pp. 3-11). Canberra: Australian College of Road Safety and Federal Office of Road Safety.

Knoblauch, R. L., Pietrucha, M. T., Nitzburg, M., 1996. Field studies of pedestrian walking speed and start-up time. Transportation Research Record, 1538, 27-38.

Lemaire, P., Bherer, L., 2005. Psychologie du vieillissement. Une approche cognitive. Bruxelles : De Boeck Université.

Lindenberger, U., Marsiske, M., Baltes, P.B., 2000. Memorizing while walking: Increase in dual-task costs from young adulthood to old age. Psychology and Aging, 15, 417-436.

Lobjois, R., Cavallo, V., 2007. Age-related differences in street-crossing decisions: The effects of vehicle speed and time constraints on gap selection in an estimation task. Accident Analysis \& Prevention, 39, 934-943.

Lobjois, R., Cavallo, V., 2009. The effects of aging on street-crossing behavior: from estimation to actual crossing. Accident Analysis \& Prevention, 41, 259-267.

Logan, G.D., Zbrodoff, N.J., 1979. When it helps to misled: Facilitative effects of increasing the frequency of conflicting stimuli in a Stroop-like Task. Memory \& Cognition, 7, 166174. 
Lord, S., Howe, T., Greenland, J., Simpson, L., Rochester, L., 2011. Gait variability in older adults: A structured review of testing protocol and clinimetric properties. Gait and Posture, 34, 443- 450.

Naveteur, J., Delzenne, J., Sockeel, P., Watelain, E., Dupuy, M.A., 2013. Crosswalk time estimation and time perception: an experimental study among older female pedestrians. Accident Analysis \& Prevention, 60, 42-49.

Observatoire National Interministériel de Sécurité Routière (ONISR), 2012. La sécurité routière en France : Bilan de l’année 2011. Paris : La Documentation Française.

Owsley, C., 2011. Aging and vision. Vision Research, 51, 367-378.

Owsley, C., McGwin, G. Jr., 2004. Association between visual attention and mobility in older adults. Journal of the American Geriatrics Society, 52, 1901-1906.

Owsley, C. McGwin, G. Jr., 2010. Vision and driving. Vision Research, 50, 2348-2361.

Oxley, J.A., Fildes, B.N., Ihsen, E., Charlton, J.L., Day, R.H., 1997. Differences in traffic judgements between young and old adult pedestrians. Accident Analysis \& Prevention, 29, 839-847.

Oxley, J.A., Ihsen, E., Fildes, B.N., Charlton, J.L., Day, R.H., 2005. Crossing roads safely: An experimental study of age differences in gap selection by pedestrians. Accident Analysis and Prevention, 37, 962-971.

Prince, F., Corriveau, H., Hébert, R., Winter, D., 1997. Gait in the elderly. Gait and Posture, $5,128-135$.

Reitan R., 1958. Validity of the trail making test as an indicator of organic brain damage. Perceptual and Motor Skills, 8, 271-276.

Salzman, B., 2010. Gait and balance disorders in older adults. American Family Physician, 82, 61-68. 
Salthouse, T.A., 1996. The processing-speed theory of adult age differences in cognition. Psychology and Aging, 103, 403-428.

Salthouse, T.A., Atkinson, T.M., Berish, D.E., 2003. Executive functioning as a potential mediator of age-related cognitive decline in normal adults. Journal of Experimental Psychology, 4, 566-594.

Schaie, K.W., 1989. Individual differences in rate of cognitive change in adulthood. In V. L. Bengston, \& K. W. Schaie (Eds.), The course of latter life: Research and reflections (pp. 68-83). New York: Springer.

Scheffer, A.C., Schuurmans, M.J., Van Dijk, N., Van der hooft, T., de Rooij, S.E., 2008. Fear of falling: measurement strategy, prevalence, risk factors and consequences among older persons. Age and Ageing, 37, 19-24.

Sheridan, P.L., Hausdorff, J.M., 2007. The role of higher-level cognitive function in gait: executive dysfunction contributes to fall risk in Alzheimer's disease. Dementia and Geriatric Cognitive Disorders, 24, 125-137.

Shinar, D., Scheiber, F., 1991. Visual requirements for safety and mobility of olderdrivers. Human Factors, 33, 507-519.

Shkuratova, N., Morris, M.E., Huxham, F., 2004. Effects of age on balance control during walking. Archives of Physical Medicine and Rehabilitation, 85, 582-8.

Snowden, R.J., Kavanagh, E., 2006. Motion perception in the aging visual system: minimum motion, motion coherence, and speed discrimination thresholds. Perception, 35, 9-24. Spirduso, W.W., Francis, K.L., MacRae, P.G., 2005. Physical dimensions of aging (2nd). Champaign, Ill.; United States: Human Kinetics Publishers.

Szumilas, M, 2010. Explaining odds ratios. Journal of the Canadian Academy of Child and Adolescent Psychiatry, 19, 227-229. 
Verhaeghen, P., Cerella, J., 2002. Aging, executive control, and attention: A review of metaanalyses. Neuroscience and Biobehavioral Reviews, 26, 849-857.

Woollacott, M. H., Shumway-Cook, A., 2002. Attention and the control of posture and gait: a review of an emerging area of research. Gait and Posture, 16, 1-14.

Woollacott, M.H., Tang, P.F., 1997. Balance control during walking in the older adult: Research and its implications. Physical Therapy, 6, 646-660.

Zegeer, C.V., Seiderman, C., Lagerwey, P.A., Cynecki, M., Ronki, M., Schneider, R., 2002. Pedestrian facilities users guide : providing safety and mobility. U.S. Departement of Transportation, Highway safety research center, University of North Carolina. http://katana.hsrc.unc.edu/cms/downloads/PedFacility_UserGuide2002.pdf

Zhuang, X., Wu, C., 2011. Pedestrians' crossing behaviors and safety at unmarked roadway in China. Accident Analysis \& Prevention, 43, 1927-1936.

Zhuang, X., Wu, C., 2012. The safety margin and perceived safety of pedestrians at unmarked roadway. Transportation Research Part F: Traffic Psychology and Behaviour, 15, 119131.

Zimmermann, P., Fimm, B., 2010. Tests d'Évaluation de l'Attention (TEA). Version 2.2. Herzogenrath: Psytest. 


\section{Figure Captions}

Fig. 1. Schematic view of the street-crossing simulator and the proposed trials
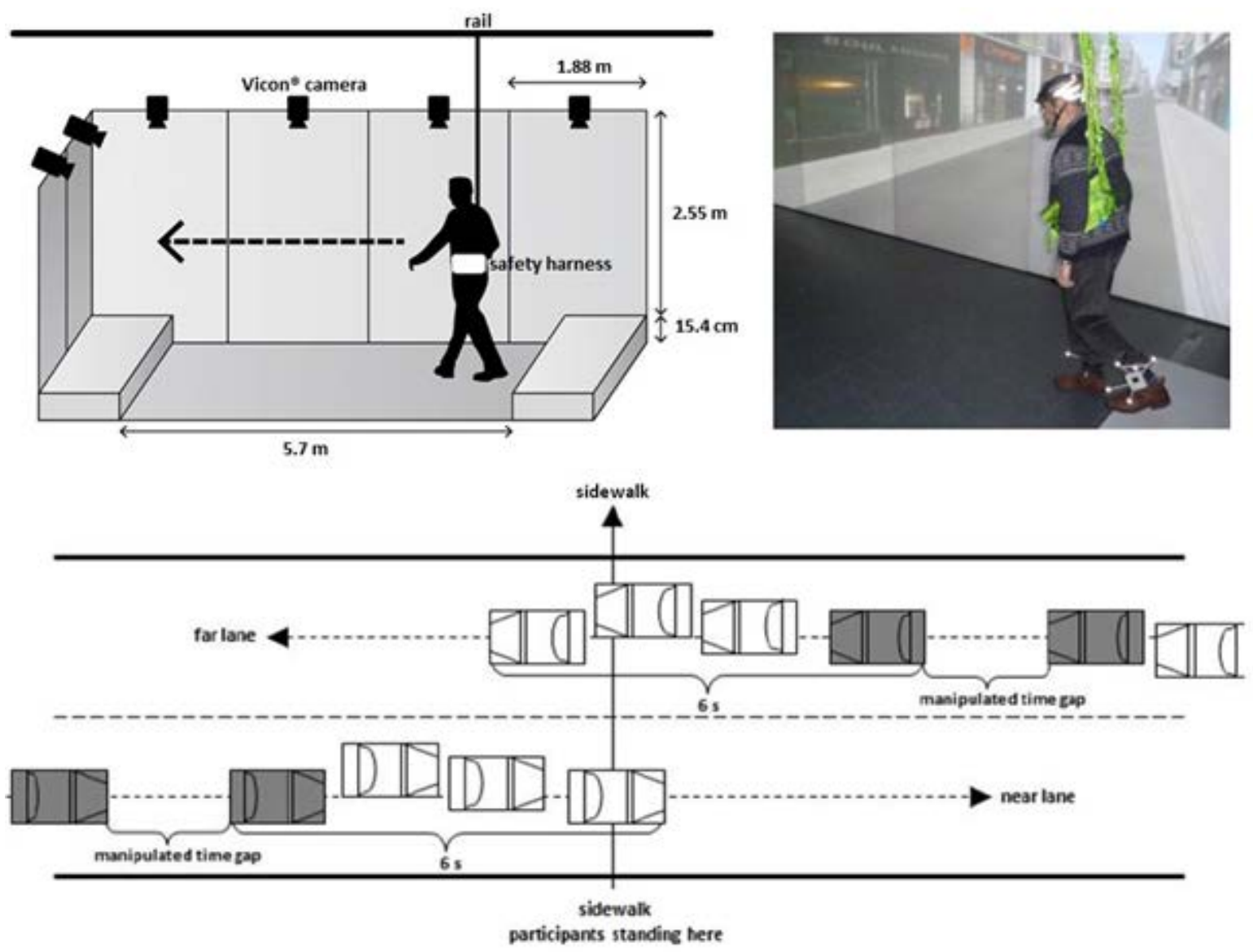
Fig. 2. Mean percentage of collisions as a function of age group. Vertical bars represent standard deviations

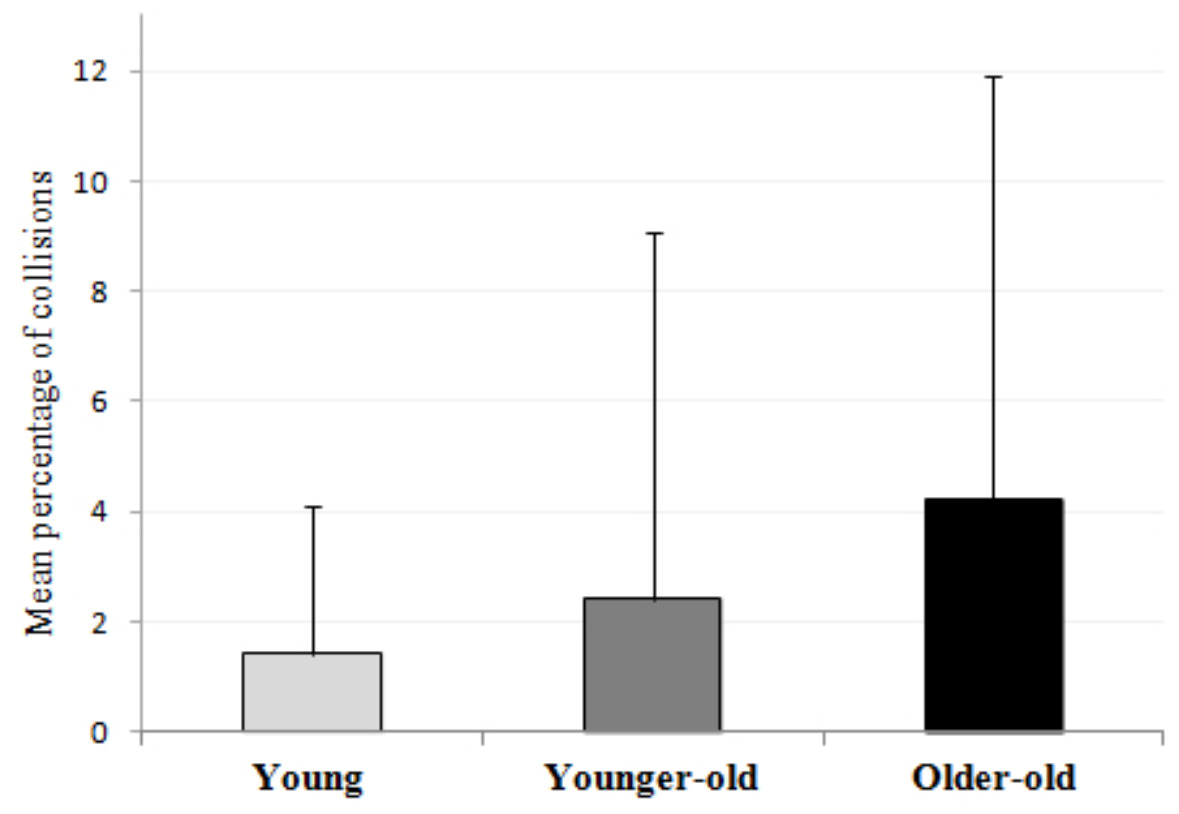


Fig. 3. Mean percentage of collisions as a function of age groups and traffic situations (a), lanes (b) and speeds of approaching cars (c). Vertical bars represent standard deviations
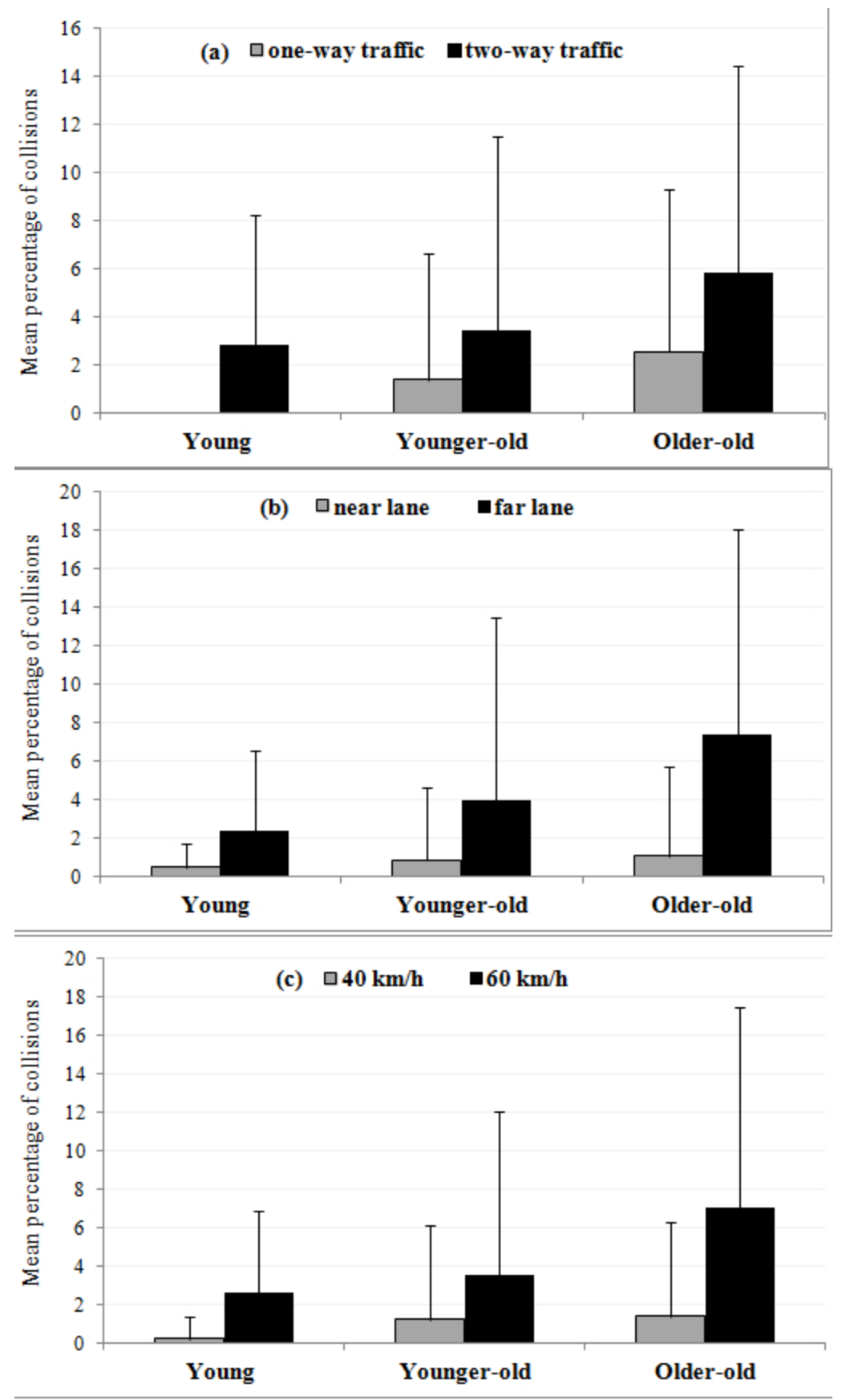
Table 1

Mean scores for visual and cognitive tests (and standard deviations) as a function of age groups

\begin{tabular}{lccc}
\hline & Young & Younger-Old & Older-old \\
\hline Visual acuity (score/12) & 11.3 & 9.84 & 8.45 \\
& $(1.75)$ & $(2.43)$ & $(2.88)$ \\
Speed of processing (threshold in ms) & 17 & 22.64 & 36.42 \\
& $(0)$ & $(17.43)$ & $(33.58)$ \\
Divided attention (threshold in ms) & 17.45 & 118.41 & 196.10 \\
Selective attention (threshold in ms) & $(1.47)$ & $(109.34)$ & $(138.99)$ \\
Shifting (cost in ms) & 100.85 & 284.86 & 363.55 \\
Inhibiting (cost in ms) & $(44.42)$ & $(95.93)$ & $(106.71)$ \\
& 110.16 & 444.58 & 521.81 \\
Updating (correct answers /15) & $(96.75)$ & $(287.76)$ & $(394.77)$ \\
& 25.79 & 86.67 & 121.34 \\
& $(36.87)$ & $(59.80)$ & $(79.55)$ \\
& 13.32 & 8.63 & 5.43 \\
\hline
\end{tabular}


Table 2

Gait parameters (and standard deviations)

\begin{tabular}{lccc}
\hline & Young & Younger-old & Older-old \\
\hline Step length (cm) & 80.74 & 67.60 & 63.64 \\
& $(4.86)$ & $(10.42)$ & $(8.12)$ \\
Step length/ height ratio & 0.4822 & 0.4026 & 0.3927 \\
& $(0.233)$ & $(0.0555)$ & $(0.0483)$ \\
\hline Step length variability (\%) & 11.15 & 13.31 & 13.62 \\
& $(1.45)$ & $(5.17)$ & $(3.18)$ \\
\hline
\end{tabular}




\section{Table 3}

Results of the hierarchical logistic regression analysis. Note: ${ }^{*} \mathrm{p} \leq .05$

\begin{tabular}{|c|c|c|c|c|c|}
\hline Step & & $\begin{array}{l}\text { Hosmer- } \\
\text { Lemeshow }\end{array}$ & & OR & $\begin{array}{l}95 \% \text { interval } \\
\text { confidence }\end{array}$ \\
\hline \multirow[t]{8}{*}{1} & $\chi 2(7)=29.6, p<.001$ & 0.178 & Visual and cognitive abilities & & \\
\hline & & & Visual acuity & $0.917 *$ & $0.849-0.989$ \\
\hline & & & Speed of processing & $1.008 *$ & $1.001-1.016$ \\
\hline & & & Divided attention & 1.000 & $0.997-1.002$ \\
\hline & & & Selective attention & $1.003^{*}$ & $1.001-1.005$ \\
\hline & & & Shifting & 1.000 & $1-1.001$ \\
\hline & & & Inhibiting & 0.997 & $0.993-1.001$ \\
\hline & & & Updating & 1.016 & $0.955-1.081$ \\
\hline \multirow[t]{11}{*}{2} & $\chi 2(2)=5.5, p<.05$ & 0.150 & Visual and cognitive abilities & & \\
\hline & & & Visual acuity & $0.919 *$ & $0.850-0.994$ \\
\hline & & & Speed of processing & 1.014* & $1.005-1.022$ \\
\hline & & & Divided attention & 0.999 & $0.997-1.002$ \\
\hline & & & Selective attention & 1.002 & $0.999-1.005$ \\
\hline & & & Shifting & 1.000 & $1-1.001$ \\
\hline & & & Inhibiting & 0.997 & $0.993-1.001$ \\
\hline & & & Updating & 1.024 & $0.961-1.090$ \\
\hline & & & Gait parameters & & \\
\hline & & & Step length & $0.003^{*}$ & $0-0.429$ \\
\hline & & & Step length variability & 0.951 & $0.884-1.024$ \\
\hline \multirow[t]{12}{*}{3} & $\chi 2(1)=0.12, p=.729$ & 0.669 & & & \\
\hline & & & $\begin{array}{l}\text { Visual and cognitive abilities } \\
\text { Visual acuity }\end{array}$ & $0.916 *$ & $0.846-0.993$ \\
\hline & & & Speed of processing & $1.014 *$ & $1.005-1.023$ \\
\hline & & & Divided attention & 0.999 & $0.997-1.002$ \\
\hline & & & Selective attention & 1.002 & $0.999-1.005$ \\
\hline & & & Shifting & 1.000 & $1-1.001$ \\
\hline & & & Inhibiting & 0.997 & $0.993-1.001$ \\
\hline & & & Updating & 1.024 & $0.961-1.091$ \\
\hline & & & Gait parameters & & \\
\hline & & & Step length & $0.002 *$ & $0-0.421$ \\
\hline & & & Step length variability & 0.950 & $0.882-1.024$ \\
\hline & & & Age & 0.997 & $0.978-1.016$ \\
\hline
\end{tabular}

